\title{
On the root mean square quantitative chirality and quantitative symmetry measures
}

\author{
Michel Petitjean ${ }^{\mathrm{a})}$ \\ ITODYS (CNRS, ESA 7086), 1 rue Guy de la Brosse, 75005 Paris, France
}

(Received 22 February 1999; accepted for publication 22 March 1999)

The properties of the root mean square chiral index of a $d$-dimensional set of $n$ points, previously investigated for planar sets, are examined for spatial sets. The properties of the root mean squares direct symmetry index, defined as the normalized minimized sum of the $n$ squared distances between the vertices of the $d$-set and the permuted $d$-set, are compared to the properties of the chiral index. Some most dissymetric figures are analytically computed. They differ from the most chiral figures, but the most dissymetric 3-tuples and the most chiral 3-tuples have a common remarkable geometric property: the squared lengths of the sides are each equal to three times a squared distance vertex to the mean point. (C) 1999 American Institute of Physics. [S0022-2488(99)01009-9]

\section{INTRODUCTION}

Chirality and symmetry properties of a solid body can be viewed as a continuous varying quantity taking values over [0;1] rather than a logical property, i.e., the body is or is not symmetric or chiral. The use of a chirality measure seems to be introduced by Rassat. ${ }^{1}$ Then, various quantitative chirality or symmetry measures have been used. ${ }^{2-12}$ This concept has received applications in physics, proposed mostly by the Avnir group. ${ }^{2-4}$

The root mean square chiral index CHI of a $d$-dimensional set of $n$ points was defined ${ }^{12}$ as the sum of the $n$ squared distances between the vertices of the set and those of its inverted image, normalized to $4 T / d, T$ being the inertia of the set. This index is computed after minimization of the sum of the squared distances in respect to all rotations and translations and all permutations between equivalent vertices. It was shown to be a second kind of continuous chirality measure taking values over $[0 ; 1]$, the zero value corresponding to an achiral compound perfectly superposed to its inverted image. Similarly, the direct symmetry index DSI of a $d$-dimensional set of $n$ points is defined here as follows. When all vertices are unequivalent, DSI is undefined. When there are at least two equivalent vertices, the sum of the $n$ squared distances between the vertices and those of the permuted set is minimized for all rotations and translations and permutations (excluding the identity permutation) between equivalent vertices. DSI is the ratio of this minimized sum to twice the inertia $T$ of the set.

The quantitative symmetry and chirality concepts used here are fully different from those of Avnir et al. for the following reasons: no achiral reference is needed to compute CHI, no symmetry assumptions are needed to compute CHI and DSI, no folding and unfolding process ${ }^{5}$ are needed here, the normalization are different, and the farthest point from the centroid is not needed here, and, of course, the extremal figures are different.

The properties of CHI were examined for monodimensional sets and planar sets. ${ }^{12}$ They are now examined for spatial sets. Hyperspatial sets (i.e., $d$ is any positive integer) are examined when all vertices are unequivalent. The major difference between planar, spatial, and hyperspatial sets lies in the expression of the optimal rotation. The properties of DSI are also examined. For clarity, a set of $n=3$ points will be called a triangle. The most dissymetric triangles, i.e., those maximizing DSI, are here analytically computed when there are two or three equivalent vertices.

${ }^{a)}$ Phone: 33 (0)1 4427 4857; fax: 33 (0)1 4427 6814; electronic mail: Petitjean@itodys.jussieu.fr 


\section{NOTATIONS}

The notations are those used in Ref. 12. X0 and X1 are the $n$ rows and $d$ columns arrays of coordinates. $\mathrm{X} 0$ is the fixed set and $\mathrm{X} 1$ is to move. The quote denotes the transposition operator. All vectors are written as one-column matrices. $\langle x \mid y\rangle$ is the scalar product of the vectors $x$ and $y$, and when $d=3, x \wedge y$ is their cross product. The trace and the determinant operators are denoted, respectively, Tr and Det. Y1 is the rotated and translated image of $\mathrm{X} 1$, and $D^{2}=\operatorname{Tr}((\mathrm{X} 0-\mathrm{Y} 1)$ $\left.\cdot(\mathrm{X} 0-\mathrm{Y} 1)^{\prime}\right)$ is the sum of the squared distances. $D^{2}$ is minimized for rotation plus translation when $\mathrm{X} 0$ and $\mathrm{X} 1$ are centered before computing the optimal rotation. Translations will be no longer considered, and the centering condition will not be assumed unless otherwise mentioned. The following matrices are used: $\mathrm{V} 00=\mathrm{X} 0^{\prime} \cdot \mathrm{X} 0, \mathrm{~V} 11=\mathrm{X} 1^{\prime} \cdot \mathrm{X} 1, \mathrm{~V} 10=\mathrm{X} 1^{\prime} \cdot \mathrm{X} 0, \mathrm{~V} 01=\mathrm{V} 10^{\prime}$, and $T=(\mathrm{T} 0+\mathrm{T} 1) / 2, \mathrm{~T} 0=\operatorname{Tr}(\mathrm{V} 00)$ and $\mathrm{T} 1=\operatorname{Tr}(\mathrm{V} 11)$ being the respective inertia of $\mathrm{X} 0$ and $\mathrm{X} 1$, reducing to the usual inertia when the arrays are centered. The identity matrix is $\mathbf{I}$, and $R$ is a rotation matrix, such that $\mathrm{Y} 1=\mathrm{X} 1 \cdot R^{\prime}$.

The correspondence between $\mathrm{X} 0$ and $\mathrm{X} 1$ is handled via an $n$-dimensional square permutation matrix $P$. Let be $\mathrm{Z} 1=P \cdot \mathrm{Y} 1$. When $\mathrm{X} 1$ is the inverted image of $\mathrm{X} 0$ and when the centering condition is satisfied, the chiral index of a spatial set is $\mathrm{CHI}=D^{2} /(4 T / d)$, with $D^{2}=\operatorname{Tr}((\mathrm{X} 0$ $\left.\left.-P \cdot \mathrm{X} 1 \cdot R^{\prime}\right) \cdot\left(\mathrm{X} 0-P \cdot \mathrm{X} 1 \cdot R^{\prime}\right)^{\prime}\right)$ being minimized over all rotations $R$ and allowed permutations $P$. When $\mathrm{X} 1$ is a rotated and translated image of $\mathrm{X} 0$ and when the centering condition is satisfied, $\mathrm{DSI}=D^{2} / 2 T, D$ being minimized over all rotations and allowed nonidentity permutations.

The computation of either CHI or DSI requires the optimal rotation superimposing two sets. When $d=3$, the analytical expression of the optimal rotation superposing $\mathrm{X} 1$ on $\mathrm{X} 0$, X0 and $\mathrm{X} 1$ being any $n$ rows and 3 columns arrays of coordinates, is given in the Appendix.

\section{THE OPTIMAL ROTATION FOR 3D ENANTIOMERS}

In this section, the centering condition is not assumed and three-dimensional enantiomers are considered. For clarity, $\mathrm{X} 0$ is noted $\sim X$ and its inverted image is $\mathrm{X} 1=-P \cdot X$, and we define $V=X^{\prime} \cdot P \cdot X=-\mathrm{V} 01$. From Appendix A, we have

$$
D^{2}=D 0^{2}-2\langle q \mid B q\rangle,
$$

the optimal quaternion $q$ being the eigenvector associated to L1, the highest eigenvalue of $B$ :

$$
\begin{gathered}
B=\left(\begin{array}{ll}
0 & c^{\prime} \\
c & A
\end{array}\right), \\
A=\operatorname{Tr}\left(V+V^{\prime}\right) \cdot \mathbf{I}-\left(V+V^{\prime}\right), \\
c=\left(\begin{array}{c}
\mathrm{V}(2,3)-\mathrm{V}(3,2) \\
\mathrm{V}(3,1)-\mathrm{V}(1,3) \\
\mathrm{V}(1,2)-\mathrm{V}(2,1)
\end{array}\right) .
\end{gathered}
$$

When $P$ is a symmetric permutation, $c$ is null, and the eigenvalues of $B$ are the three eigenvalues of $A$ and zero.

\section{ENANTIOMERS WITH ALL VERTICES UNEQUIVALENT}

All the conditions of the preceding section are assumed to stand, and the vertices are all unequivalent, i.e., the only allowed permutation is $P=\mathbf{I} . V=X^{\prime} \cdot X$ is symmetric and $c$ is therefore null. The sum of squares prior rotation is $\mathrm{D}^{2}=4 \operatorname{Tr}(V)$, which is the maximized $D^{2}$ value because zero is the smallest eigenvalue of $B$. We have $A=2(\operatorname{Tr}(V) \cdot \mathbf{I}-V)$. Let v1, v2, v3 be the eigenvalues of $V$ arranged in decreasing order. The largest eigenvalue of $B$ is $\mathrm{L} 1=\mathrm{d} 1=2(\mathrm{v} 1+\mathrm{v} 2)$ and the optimal rotation of $-X$ is 180 degrees around the principal axis associated to the smallest eigenvalue of $V$. Now we have $D^{2}=4 \operatorname{Tr}(V)-4(\mathrm{v} 1+\mathrm{v} 2)$, i.e., 


$$
D^{2}=4 \mathrm{v} 3 .
$$

We assume now that $X$ is centered, i.e., $V$ is $n$ times its variance matrix. The chiral index of the set of $n$ vertices is therefore:

$$
\mathrm{CHI}=3 \mathrm{v} 3 /(\mathrm{v} 1+\mathrm{v} 2+\mathrm{v} 3) .
$$

$\mathrm{CHI}$ is $d$ times the percentage of inertia associated to the smallest eigenvalue of $V$. Looking at Eqs. (3) and (7) in Ref. 12 and Appendix 1 in Ref. 12, we can see that this is also true for planar sets $(d=2)$ and unidimensional sets $(d=1)$.

The eigenvalues of $V$ being positive and in decreasing order, $\mathrm{CHI}$ is maximized for $\mathrm{v} 1=\mathrm{v} 2=\mathrm{v} 3=\mathrm{v}$, i.e., $\mathrm{CHI}=1$ and $V=\mathrm{v} \cdot \mathbf{I}$. When $X$ has only 4 vertices, it is therefore a regular tetrahedron (see Appendix 2 in Ref. 12).

\section{HYPERSPATIAL SETS WITH ALL UNEQUIVALENT VERTICES}

The optimal rotation superimposing two $d$-dimensional sets is unknown when $d>3$, except for enantiomers with all unequivalent vertices, as shown hereafter. The sum of squares to be minimized is $D^{2}=\operatorname{Tr}\left(\left(X-X \cdot Q^{\prime}\right) \cdot\left(X-X \cdot Q^{\prime}\right)^{\prime}\right)=2\left(\operatorname{Tr}\left(X^{\prime} \cdot X\right)-\operatorname{Tr}\left(Q \cdot X^{\prime} \cdot X\right)\right), X$ being the $(n, d)$ array of coordinates and $Q$ being an orthogonal matrix with $\operatorname{det}(Q)=-1$. Thus, $\operatorname{Tr}\left(Q \cdot X^{\prime} \cdot X\right)$ has to be maximized. Assuming that $X$ is in its principal components axis (i.e., $V=X^{\prime} \cdot X$ is diagonal), we have to find the maximum of $E=\mathrm{v}(1) \cdot \mathrm{Q}(1,1)+\mathrm{v}(2) \cdot \mathrm{Q}(2,2)+\ldots+\mathrm{v}(d) \cdot \mathrm{Q}(d, d), \mathrm{v}(1), \ldots, \mathrm{v}(d)$ being the eigenvalues of $V$ in decreasing order.

$$
\begin{aligned}
E= & {[(\mathrm{v}(1)-\mathrm{v}(d)) \cdot \mathrm{Q}(1,1)+(\mathrm{v}(2)-\mathrm{v}(d)) \cdot \mathrm{Q}(2,2)+\cdots+(\mathrm{v}(d-1)-\mathrm{v}(d))} \\
& \cdot \mathrm{Q}(d-1, d-1)]+\mathrm{v}(d) \cdot \operatorname{Tr}(Q) .
\end{aligned}
$$

The eigenvalues of $Q$ can be either +1 , or -1 , or pairs of conjugate complex roots of 1 . It follows that $\operatorname{Tr}(Q)$ is maximized when $d-1$ eigenvalues are +1 and one is -1 . Obviously, the sum of the $d-1$ terms $(\mathrm{v}(i)-\mathrm{v}(d)) \cdot Q(i, i)$ is also maximized for $\mathrm{Q}(i, i)=1$ when $i<d$. Thus $E$ is maximized and $D^{2}$ is minimized when $X$ and its enantiomer have opposite coordinates on the principal axis with smallest inertia. Thus, Eqs. (5) and (6) are generalized:

$$
D^{2}=4 \mathrm{v}(d),
$$

and assuming $X$ centered:

$$
\mathrm{CHI}=d \cdot \mathrm{v}(d) /(\mathrm{v}(1)+\mathrm{v}(2)+\cdots+\mathrm{v}(d)) .
$$

As previously, $\mathrm{CHI}$ is maximized when all eigenvalues of $V$ are equal. When $n=d+1$, CHI is therefore maximized when $X$ is a regular $d$-simplex. (See Appendix 2 in Ref. 12.)

From Eq. (8), it is possible to compare practical CHI values with the distribution of CHI when $X$ is an isotropic multinormal sample. $V$ is a Wishart matrix,${ }^{13}$ from which the joint density of the percentages of inertia can be derived, ${ }^{14}$ leading to the distribution ${ }^{15}$ of $\mathrm{CHI} / d$. Unfortunately, the final expression is not trivial when $d>2$.

\section{THE DIRECT SYMMETRY INDEX}

In this section, $d$-dimensional sets are considered and the centering condition is not assumed. The situation where all vertices are unequivalent precludes the existence of direct symmetry in the set. This situation should not be confused with the purely geometric situation where all vertices are equivalent (i.e., undistinct), for which symmetry properties are potentially observable. Thus, we consider now only sets with at least two equivalent vertices. As for the chiral index, the sum $D^{2}$ 
of the $n$ squared distances between the vertices and those of the permuted set is minimized for all rotations and all authorized permutations, excluding of course the identity permutation $P=\mathbf{I}$. When the set is centered DSI $=D^{2} /(2 T)$.

$P$ being fixed, the sum of the squared distances to minimize is, as previously, $D^{2}=\operatorname{Tr}((\mathrm{X} 0$ $\left.\left.-P \cdot \mathrm{X} 1 \cdot R^{\prime}\right) \cdot\left(\mathrm{X} 0-P \cdot \mathrm{X} 1 \cdot R^{\prime}\right)^{\prime}\right)$. Setting $\mathrm{X} 0=\mathrm{X} 1=X$ and $V=X^{\prime} \cdot P \cdot X$, we get

$$
D^{2}=2\left(T-\operatorname{Tr}\left(V \cdot R^{\prime}\right)\right) .
$$

There are at least two equivalent points $x$ and $y$. Thus the minimum of $D^{2}$ for all rotations and permutations cannot exceed the minimum of $D^{2}$ for all rotations and for the permutation exchanging $x$ and $y$, i.e., $P$ is such that $V=x \cdot y^{\prime}+y \cdot x^{\prime}+Z^{\prime} \cdot Z$, with $Z$ being the $(n-2, d)$ block extracted from $X$ by elimination of $x$ and $y$. For this permutation,

$$
\operatorname{Tr}\left(V \cdot R^{\prime}\right)=y^{\prime} \cdot R^{\prime} \cdot x+x^{\prime} \cdot R^{\prime} \cdot y+\operatorname{Tr}\left(Z^{\prime} \cdot Z \cdot R^{\prime}\right) .
$$

Assuming $d>1$, a rotation exists $R$ which rotates from +90 degrees the first axis toward the second axis, i.e., $R(2,1)=-R(1,2)=1, R(i, i)=1$ for $i>2$, all other elements of $R$ being null. Thus, $R+R^{\prime}=0, \quad y^{\prime} \cdot R^{\prime} \cdot x+x^{\prime} \cdot R^{\prime} \cdot y=0$ and $-\operatorname{Tr}\left(Z^{\prime} \cdot Z \cdot R^{\prime}\right)=\operatorname{Tr}\left(Z^{\prime} \cdot Z \cdot R\right)=\operatorname{Tr}\left(R^{\prime} \cdot Z^{\prime} \cdot Z\right)$ $=\operatorname{Tr}\left(Z^{\prime} \cdot Z \cdot R^{\prime}\right)=0$, which means that $\operatorname{Tr}\left(V \cdot R^{\prime}\right)$ is null. Because a permutation and a rotation exist such that $\operatorname{Tr}\left(V \cdot R^{\prime}\right)=0$, it follows from (9) that the minimum of $D^{2}$ is upper bounded by $2 T$, and then DSI pertains to $[0 ; 1]$ when $d>1$.

The following centered set containing three points is such that DSI $=1$ for all $d>1: x=e 1$ $\cdot(-1-\sqrt{3}) / 2, y=e 1 \cdot(-1+\sqrt{3}) / 2, z=e 1, e 1$ being the first base vector, $x$ and $y$ being equivalent and $z$ being not.

When $d=1, x$ and $y$ are numbers, $Z$ is a vector, $T=x^{2}+y^{2}+Z^{\prime} \cdot Z, R=1$, and $\operatorname{Tr}\left(V \cdot R^{\prime}\right)$ $=2 x \cdot y+Z^{\prime} \cdot Z$. Thus, $4 T-D^{2}=2 \cdot\left(T+\operatorname{Tr}\left(V \cdot R^{\prime}\right)\right)=2(x+y)^{2}+4 \cdot Z^{\prime} \cdot Z$, which cannot be negative. Thus, for $d=1, D^{2}$ varies from 0 to $4 T$ and the direct symmetry index pertains to [0;2], the extremal value DSI $=2$ being reached for a centered set containing two opposite values. But of course, direct rotational symmetry has little interest for $d=1$.

Computing simultaneously CHI and DSI for spatial sets is easy, since they both lead to the same quadratic form defined by Eqs. (1)-(4), except that the quadratic form associated to DSI now takes the opposite sign, because $\mathrm{X} 1$ was set to $X$ rather than to $-X$. It means that the smallest eigenvalue L4 should be used to compute DSI rather than L1 for CHI, the minimized sum of squared distances being now

$$
D^{2}=\mathrm{D} 0^{2}+2 \mathrm{~L} 4
$$

As shown in the Appendix, L4 is always nonpositive. Another difference between CHI and DSI is that the normalizing coefficients are, respectively, $4 T / d$ and $2 T$, but this is not a crucial difference.

\section{THE DIRECT SYMMETRY INDEX OF PLANAR TRIANGLES}

We assume that $d=2$. Let $x$ be the column vector of the abscissas, and $y$ the column vector of their ordinates: $x^{\prime}=\left(x_{1}, x_{2}, \ldots, x_{n}\right)$ and $y^{\prime}=\left(y_{1}, y_{2}, \ldots, y_{n}\right)$. The points will be $p_{1}, p_{2}, \ldots, p_{n}$. The image of $(x, y)$ through the permutation $P$ is $(P x, P y)$. $P$ being fixed, the distance $D$ minimized for all rotations is known: ${ }^{12}$

$$
D^{2}=2(T-E),
$$

$E$ being the non-negative number, such that

$$
E^{2}=\left(x^{\prime} P^{\prime} x\right)^{2}+\left(x^{\prime} P^{\prime} y\right)^{2}+\left(y^{\prime} P^{\prime} x\right)^{2}+\left(y^{\prime} P^{\prime} y\right)^{2}+2\left(x^{\prime} P^{\prime} x\right)\left(y^{\prime} P^{\prime} y\right)-2\left(y^{\prime} P^{\prime} x\right)\left(x^{\prime} P^{\prime} y\right) .
$$

Thus, 


$$
E^{2}=\left(x^{\prime} P x+y^{\prime} P y\right)^{2}+\left(y^{\prime} P x-x^{\prime} P y\right)^{2} .
$$

The minimization for rotations plus translations is reached when the set is centered. The inertia is thus $T=x^{\prime} x+y^{\prime} y$. We assume $T$ non-null, i.e., there are at least two distinct points. Let $\mathbf{1}$ be the $n$-vector such that all its $n$ components are 1 . Centering means $\mathbf{1}^{\prime} x=\mathbf{1}^{\prime} y=0$. We define also $M$ $=\left(P+P^{\prime}\right) / 2$ and $N=\left(P-P^{\prime}\right) / 2$, which implies that $x^{\prime} N x=y^{\prime} N y=0$.

We assume now that $n=3$, and that all vertices are equivalents. $d_{12}, d_{23}$ and $d_{31}$ are the respective lengths of the sides of the triangle. $\left\|p_{1}\right\|,\left\|p_{2}\right\|$ and $\left\|p_{3}\right\|$ are the lengths of the segments joining the barycenter to the vertices at the opposite of the sides with respective lengths $d_{23}, d_{31}$ and $d_{12}$. The inertia can be also written as $T=\left\|p_{1}\right\|^{2}+\left\|p_{2}\right\|^{2}+\left\|p_{3}\right\|^{2}$, or $T=\left(d_{23}^{2}+d_{31}^{2}+d_{12}^{2}\right) / 3$. $\left.+d_{31}^{4}\right)$.

The surface $S$ of the triangle is such that $16 S^{2}=2\left(d_{12}^{2} d_{23}^{2}+d_{23}^{2} d_{31}^{2}+d_{31}^{2} d_{12}^{2}\right)-\left(d_{12}^{4}+d_{23}^{4}\right.$

\section{A. Extremal values for a given permutation}

Using $M$ and $N$, Eq. (13) becomes

$$
E^{2}=\left(x^{\prime} M x+y^{\prime} M y\right)^{2}+4\left(x^{\prime} N y\right)^{2} .
$$

The gradient of $\left(1-D^{2} / 2 T\right)^{2}=\left(E^{2} / T^{2}\right)$ if set to zero for $x$, then for $y$,

$$
\begin{aligned}
& T\left(x^{\prime} M x+y^{\prime} M y\right) M x+2 T\left(x^{\prime} N y\right) N y=E^{2} x, \\
& T\left(x^{\prime} M x+y^{\prime} M y\right) M y-2 T\left(x^{\prime} N y\right) N x=E^{2} y .
\end{aligned}
$$

Multiplying on the left (15) by $x^{\prime}$ then (16) by $y^{\prime}$, and substracting,

$$
T\left(x^{\prime} M x\right)^{2}-T\left(y^{\prime} M y\right)^{2}=E^{2}\left(x^{\prime} x-y^{\prime} y\right) .
$$

Then from (15) or (16),

$$
T\left(x^{\prime} M x+y^{\prime} M y\right)\left(x^{\prime} M y\right)=E^{2}\left(x^{\prime} y\right) .
$$

From (17) and (18), it comes

$$
E^{2}\left(x^{\prime} M y\right)\left(x^{\prime} x-y^{\prime} y\right)=E^{2}\left(x^{\prime} y\right)\left(x^{\prime} M x-y^{\prime} M y\right)
$$

When $n=3,5$ permutations are possible: 3 are symmetric and 2 are circular.

When $P$ is symmetric, $M=P, N=0$, and Eqs. (15) and (16) reduce to the same eigenvalues equations: $T^{2} P x=E^{2} x$ and $T^{2} P y=E^{2} y$. For $n=3$, the eigenvalues of $P$ are $+1,+1$ and -1 . Only the solution such that $E^{2}=T^{2}$ is possible, implying $D=0$, leading to a minimum for DSI, rather to a maximum.

When $P$ is one of the 2 circular permutations (the other being its transposed), we have: $2 \mathbf{M}$ $=\mathbf{1} \cdot \mathbf{1}^{\prime}-\mathbf{I}$, implying that $x^{\prime} M x=-x^{\prime} x / 2$ and $y^{\prime} M y=-y^{\prime} y / 2$, and then $E^{2}=4\left(x^{\prime} N y\right)^{2}+T^{2} / 4$. Moreover, $2 x^{\prime} N y$ is equal to the determinant of the matrix $[\mathbf{1}|x| y]$ or to the opposite of this determinant, depending on which circular permutation is used. That implies $E^{2}=4 S^{2}+T^{2} / 4$. The minimum is therefore reached by a null-area triangle: the points are aligned.

\section{B. Maximizing DSI}

Let us consider the symmetric permutation associating $p_{1}$ to itself. The following comes: $N$ $=0$ and $E^{2}=2 x_{2} x_{3}+x_{1}^{2}+2 y_{2} y_{3}+y_{1}^{2}=\left(T-d_{23}^{2}\right)^{2}$. Similarly, the $E$ values associated with the symmetric permutations associating $p_{2}$ with $p_{2}$ and $p_{3}$ with $p_{3}$ are such that $E^{2}=\left(T-d_{31}^{2}\right)^{2}$ and $E^{2}=\left(T-d_{12}^{2}\right)^{2}$. Both circular permutations lead to $E^{2}=4 S^{2}+T^{2} / 4, S^{2}$ and $T$ being homogeneous polynomials of $d_{12}^{2}, d_{23}^{2}$ and $d_{31}^{2}$. The 4 expressions of $E^{2}$ are homogeneous polynomials of 3 variables, returning non-negative values. 
For a given triangle, the optimal permutation is that which leads to the highest $E$ value. Thus a maximum of DSI, or a minimum of $\left[\operatorname{Max}\left(E^{2}\right) / T^{2}\right]$, should be searched either among the extrema of $E^{2} / T^{2}$ associated with a permutation, or at the intersection of at least two of the 4 polynomials associated with the permutations, $T$ being the same for all permutations.

It was shown above that only one extremum of $E^{2} / T^{2}$ is useful, and it is such that $E=T / 2$ and $S=0$. This is possible only if the length of a side is equal to the sum of the two others. Assuming for example, that $d_{23}=d_{31}+d_{12}$, and reporting it in $E=\left|T-d_{23}^{2}\right|=\left|d_{12}^{2}+d_{31}^{2}-2 d_{23}^{2}\right| / 3$. The following comes: $E=\left(d_{23}^{2}+2 d_{12} d_{31}\right) / 3$, or $E=(T / 2)+d_{12} d_{31}$. The circular permutation could be optimal only if $d_{12} d_{31}=0$, which should imply that DSI $=0$ (degenerate isocele triangle). Similar conclusions should be reached if $d_{31}$ or $d_{12}$ have been used: the extrema of $E^{2} / T^{2}$ associated to a given permutation are not adequate.

Thus, it is needed to look at the intersection of the polynomials. Noting that the $E / T$ values depend only on the distances ratios, we can work with only two independent variables, and we search the minimum at the intersection of 3 among the 4 polynomials. There are at least 2 among 3 symmetric permutations which lead to the same value. Assuming, for example, that $E=\mid T$ $-d_{12}^{2}|=| T-d_{31}^{2} \mid$, thus, $\left|d_{23}^{2}+d_{31}^{2}-2 d_{12}^{2}\right|=\left|d_{12}^{2}+d_{23}^{2}-2 d_{31}^{2}\right|$. Either we get $d_{12}^{2}=d_{31}^{2}$, which does not work because the triangle should be isocele $(\mathrm{DSI}=0)$, or we get $2 d_{23}^{2}=d_{31}^{2}+d_{12}^{2}$, and thus $E=\left|d_{12}^{2}-d_{31}^{2}\right| / 2$. In this situation, the $E$ value associated to the third symmetric permutation is null, and the $E$ value associated to circular permutations is $E=d_{12} d_{31}$. The equality between the 3 nonzero $E^{2}$ values give the desired relation: $\left(d_{12}^{2}-d_{31}^{2}\right)^{2}=4 d_{12}^{2} d_{31}^{2}$. Reusing $2 d_{23}^{2}=d_{31}^{2}+d_{12}^{2}$, the ratios of the squared lengths of the sides are deduced: $\left(d_{12}^{2} / d_{23}^{2}\right)=1+\sqrt{2} / 2,\left(d_{31}^{2} / d_{23}^{2}\right)=1-\sqrt{2} / 2$, and $E^{2} / T^{2}=(1-\mathrm{DSI})^{2}=1 / 2$.

\section{Remarkable geometric properties of the optimal triangles}

Using the distances, we get the angles associated, respectively, to the points $p_{1}, p_{2}$ and $p_{3}: \pi / 4, \pi / 8$ and $5 \pi / 8$.

A possible set of coordinates of the most dissymetric triangle is

$$
X=\left(\begin{array}{cc}
\sqrt{2} / 3 & 1 / 3 \\
(-3-\sqrt{2}) / 6 & -1 / 6 \\
(3-\sqrt{2}) / 6 & -1 / 6
\end{array}\right) \text {. }
$$

It is easy to see that $d_{23}^{2}=3\left\|p_{1}\right\|^{2}, d_{12}^{2}=3\left\|p_{2}\right\|^{2}$, and $d_{31}^{2}=3\left\|p_{3}\right\|^{2}$. It should be pointed out that this relation is symmetric only for $p_{2}$ and $p_{3}$. This remarkable proportionality exists also for the degenerate triangle with only two equivalent vertices, which was cited in Sec. VI, and corresponding to the maximal value $\mathrm{DSI}=1$, for any dimension $d>1$. For $d=2$, the most chiral triangles also offer this remarkable proportionality, discarding which vertices are equivalent, ${ }^{12}$ but none of them has the shape of the most dissymmetric triangle. The shape of the most dissymmetric triangle has been measured using random triangles, with vertices uniformly distributed over a square. The results (Table I) are in accordance with the theory.

\section{DISCUSSION AND CONCLUSION}

The properties of the RMS (root mean square) chiral index have been examined for spatial sets. As for planar sets, it is easily analytically computed, but the expression of the optimal 3D rotation is fully different from those of the $2 \mathrm{D}$ one. The optimal rotation is unknown for hyperspatial sets, except when $X$ is superposed with its unpermuted enantiomer. When $d>3$, it is proposed to extend the iterative procedure ${ }^{16}$ to compute the optimal rotation superposing two $d$-dimensional sets, and to use it for permuted enantiomers. Similarly, computing the RMS direct symmetry index is easy for 2D and 3D sets, but suffers from the same limitation than the chiral index when $d>3$.

Looking at Eq. (8), it is clear that the RMS chiral index is also extendible to continuous distributions with all distinct points, provided that the variance exists. When there are subsets of 
TABLE I. Measure of the shape of the most dissymmetric triangle with three equivalent vertices. Ntr: number of random triangles. Popt: optimal permutation. DSI: direct symmetry index. The 3 angles are expressed as multiples of $\pi / 8$.

\begin{tabular}{rlcc}
\hline \hline \multicolumn{1}{r|}{ Ntr } & Popt & $(1 \text {-DSI })^{2}$ & Angles \\
\hline 1 & 321 & 0.724887 & 0.8291821 .3057125 .865106 \\
11 & 312 & 0.632934 & 1.2399002 .7105874 .049513 \\
13 & 231 & 0.546920 & 1.1600931 .8594414 .980466 \\
37 & 213 & 0.539919 & 1.0041531 .8650565 .130791 \\
85 & 321 & 0.537833 & 1.1207571 .8220105 .057233 \\
179 & 321 & 0.519058 & 0.9888152 .0671364 .944049 \\
363 & 321 & 0.513820 & 0.9936752 .0546284 .951697 \\
751 & 231 & 0.503264 & 1.0074082 .0094804 .983112 \\
13052 & 213 & 0.501541 & 0.9995412 .0067784 .993681 \\
51783 & 231 & 0.500970 & 1.0014852 .0077494 .990766 \\
161448 & 231 & 0.500631 & 1.0017841 .9994544 .998762 \\
394890 & 231 & 0.500541 & 1.0007202 .0050674 .994213 \\
1097067 & 231 & 0.500420 & 1.0008292 .0020944 .997077 \\
1347455 & 312 & 0.500412 & 1.0008072 .0020924 .997100 \\
1483751 & 132 & 0.500085 & 1.0001981 .9996385 .000164 \\
62565625 & 132 & 0.500073 & 1.0002131 .9996735 .000114 \\
90476880 & 132 & 0.500062 & 1.0000122 .0003744 .999613 \\
143978185 & 321 & 0.500048 & 1.0000322 .0003694 .999599 \\
178782085 & 312 & 0.500024 & 1.0000372 .0001894 .999775 \\
\hline \hline
\end{tabular}

undistinct points, handling continuous sets is more difficult, because the set of authorized permutations must be redefined. This latter remark applies to the direct symmetry index. Thus, the extension of CHI and DSI to continuous sets will be examined in a further work.

The most chiral triangles and the most dissymmetric triangles offer the same remarkable geometric property. Its extension to higher-dimensional simplices is an open problem.

The chiral index and the direct symmetry index provides a coherent quantification of rotational symmetries carrying more information than a boolean value indicating the presence or absence of such symmetries. Although a perfect symmetry can be destroyed when a small perturbation is applied, the ability to quantify proper and improper rotational symmetries provides a robust tool to overcome this problem. As a by-product of computing CHI or DSI, the axe and angle associated to the optimal quaternion locate nonambiguously the symmetry element. When computing either CHI or DSI, if more than one permutation leads to small values of the indices, the set of optimal quaternions provides informations about the existence of more than one symmetry element. Building an automated procedure returning all symmetry elements of a perturbated symmetric set is currently investigated.

\section{ACKNOWLEDGMENT}

The author thanks Professor D. Avnir for useful discussions and encouragement to publish this work.

\section{APPENDIX: THE OPTIMAL ROTATION FOR SPATIAL SETS}

In this section, the centering condition is not assumed, and $d=3$. X0 and $\mathrm{X} 1$ are any $n$ rows and 3 columns arrays of coordinates. The identity permutation $P=\mathbf{I}$ is assumed, but the final result will be valid for any $P$ with replacing X1 by $P \cdot \mathrm{X} 1$. The well-known Procrustes algorithm ${ }^{16,17}$ used to find the optimal orthogonal transformation superposing two $d$-sets does not work for enantiomers, because it leads always to $D=0$ and the optimal orthogonal matrix has a negative determinant $($ Det $=-1)$. Some iterative procedures are available, ${ }^{18,19}$ but the final expression of the optimal rotation was found by Diamond, ${ }^{20}$ leading to express $D^{2}$ with a quadratic form of the 
quaternion associated to the rotation. This quadratic form is maximized by an orthonormal basis of four quaternions. For convenience, the expression of the optimal rotation is retrieved here following a different presentation.

A 3D-rotation $R$ is associated to a 3D rotation axis $u$ and to a rotation angle $r$. This is expressed with a quaternion $q=(p, u)$, with $p=\cos (r / 2)$ and $\|u\|=\langle u \mid u\rangle^{1 / 2}=\sin (r / 2)$. Thus $\langle q \mid q\rangle=1$, and the image of a point $x$ through $R$ is $^{21} R x=(1-2\langle u \mid u\rangle) x+2\langle u \mid x\rangle u+2 p(u \wedge x)$. Because $(-p,-u)$ is the same rotation than $(p, u), p$ is always taken non-negative, i.e., $r$ takes values from 0 to 180 degrees.

Let $c$ be the sum of the $n$ vectors $x 1_{i} \wedge \mathrm{x} 0_{i}$. Thus, we have $c(1)=\mathrm{V} 10(2,3)-\mathrm{V} 10(3,2)$, $c(2)=\mathrm{V} 10(3,1)-\mathrm{V} 10(1,3)$, and $c(3)=\mathrm{V} 10(1,2)-\mathrm{V} 10(2,1)$. The matrix $A$ is defined as $A$ $=(\mathrm{V} 10+\mathrm{V} 01)-\operatorname{Tr}(\mathrm{V} 10+\mathrm{V} 01) \cdot \mathbf{I}$. Let $\mathrm{D}^{2}$ be the initial sum of squares, prior to rotating $\mathrm{X} 1$. Now, we have the following equalities: $\left\langle\operatorname{Rx} 1_{i} \mid x 0_{i}\right\rangle=(1-2\langle u \mid u\rangle)\left\langle x 1_{i} \mid x 0_{i}\right\rangle+2\left\langle u \mid x 1_{i}\right\rangle\left\langle u \mid x 0_{i}\right\rangle$ $+2 p\left\langle u \wedge x 1_{i} \mid x 0_{i}\right\rangle=(1-2\langle u \mid u\rangle)\left\langle x 1_{i} \mid x 0_{i}\right\rangle+\left\langle u \mid\left(x 1_{i} \cdot x 0_{i}^{\prime}+x 0_{i} \cdot x 1_{i}^{\prime}\right) u\right\rangle+2 p\left\langle u \mid x 1_{i} \wedge x 0_{i}\right\rangle . \mathrm{V} 10$ is the sum of the $n$ matrices $x 1_{i} \cdot x 0_{i}^{\prime}$, and $\operatorname{Tr}(V 10)$ is the sum of the $n$ quantities $\left\langle x 1_{i} \mid x 0_{i}\right\rangle$. Thus we get $D^{2}=\mathrm{D} 0^{2}-2\langle u \mid A u\rangle-4 p\langle u \mid c\rangle$. Let us define the $4 \times 4$ matrix $B$ :

$$
B=\left(\begin{array}{ll}
0 & c^{\prime} \\
c & A
\end{array}\right),
$$

$q=(p, u)$ being the unknown quaternion; it follows that: $D^{2}=\mathrm{D} 0^{2}-2\langle q \mid B q\rangle$.

$B$ is a constant symmetric matrix depending only on the input data, and the quadratic form $\langle q \mid B q\rangle$ has to be maximized, $q$ being a unit vector. This problem has a well-known solution: ${ }^{17}$ the stationary points are an othonormal basis eigenvectors of $B$, and the associated eigenvalues are the optimal values of the quadratic form. The sense of each eigenvector is known because $p$ must be non-negative. It is unimportant to get $+u$ or $-u$ when $p=0$. Let L1, L2, L3, L4 be the eigenvalues arranged in decreasing order.

$B$ is the sum of two $4 \times 4$ symmetric matrices. One contains only $A$ and zeros on the first row and column. Let B1 be this matrix. The other contains only $c^{\prime}$ on the right of the first row and $c$ on the bottom of the first column, zero as a first diagonal element, and nine zeros in the remaining $3 \times 3$ block. Let B2 be this one-rank matrix, of which the four eigenvalues are obviously $\|c\|$ and zero with three as multiplicity. Let $\mathrm{d} 1, \mathrm{~d} 2, \mathrm{~d} 3$ be the eigenvalues of $A$ arranged in decreasing order. Thus, the following inequalities stand: ${ }^{22}$ the eigenvalues of $A$ separate those of $B: \mathrm{L} 1 \geqslant \mathrm{~d} 1 \geqslant \mathrm{~L} 2 \geqslant \mathrm{~d} 2 \geqslant \mathrm{~L} 3 \geqslant \mathrm{~d} 3 \geqslant \mathrm{~L} 4$, and $\left|\mathrm{Li}-\mathrm{di}^{\prime}\right| \leqslant\|c\|$ for $i=1,2,3,4, \mathrm{di}^{\prime}$ being the $i$ th greatest value among $(0, \mathrm{~d} 1, \mathrm{~d} 2, \mathrm{~d} 3)$.

Two situations may arise. If $\mathrm{d} 1$ and $\mathrm{d} 3$ have not the same sign, the first set of inequalities means that L1 and L4 have not the same sign. If d1 and d3 have the same sign, let us look at the determinant of $B$ expressed after diagonalization of $A$. The components of $c$ become $c(1), c(2)$, $\mathrm{c}(3)$, and $\operatorname{det}(B)=-\mathrm{c}(1)^{2} \cdot \mathrm{d} 2 \cdot \mathrm{d} 3-\mathrm{c}(2)^{2} \cdot \mathrm{d} 1 \cdot \mathrm{d} 3-\mathrm{c}(3)^{2} \cdot \mathrm{d} 1 \cdot \mathrm{d} 2$. This determinant cannot be positive, thus again L1 and L4 cannot have the same sign.

Thus L1 is always non-negative and L4 is always non-positive. The rotation minimizing $D^{2}$ is those associated to the quaternion $\mathrm{q} 1$, such that $D^{2}=\mathrm{D} 0^{2}-2 \mathrm{~L} 1$, and the rotation associated to $\mathrm{q} 4$ such that $D^{2}=\mathrm{D}^{2}-2 \mathrm{~L} 4$ is that which maximizes $D^{2} . D^{2}$ has one saddle point associated to q2 and one associated to $\mathrm{q} 3$.

Some minor properties of the four optimal quaternions are obtained from their othonormality. Considering the first row of the equation $\mathrm{Bq}=\mathrm{Lq}$, it comes that $\mathrm{D} 0^{2}-D^{2}=2 \mathrm{~L}=2\langle\mathrm{v} \mid c\rangle$, with $\mathrm{v}$ $=u / p$. It shows than only a positive $L$ value leads to $D^{2}<\mathrm{D} 0^{2}$. The three others equations may be rewritten: $(A-\langle\mathrm{v} \mid n\rangle \mathbf{I}) \mathrm{v}+n=0$, but this is neither an eigenvector equation nor a linear system. Two distinct directions $u i$ and $u j$ are generally not orthogonal: $\cos (u i, u j)=-p i \cdot p j /\left(\left(1-p i^{2}\right) \cdot(1\right.$ $\left.\left.-p j^{2}\right)\right)^{1 / 2}=-\cot g(r i) \cdot \cot g(r j), r i$ and $r j$ being the 2D-angles associated, respectively, to $q i$ and $q j$.

\footnotetext{
${ }^{1}$ A. Rassat, "Un critère de classement des systèmes chiraux de points à partir de la distance au sens de Haussdorf,", Compt. Rend. Acad. Sci. Paris 299, Sér. II, 53-55 (1984).
} 
${ }^{2}$ Y. Pinto, P. W. Fowler, D. Mitchell, and D. Avnir, "Continuous chirality analysis of model Stone-Wales rearrangements in fullerenes," J. Phys. Chem. B 102, 5776-5784 (1998).

${ }^{3}$ D. R. Kanis, J. S. Wong, T. J. Marks, M. A. Ratner, H. Zabrodsky, S. Keinan, and D. Avnir, "Continuous symmetry analysis of hyperpolarizabilities. Characterization of the second-order nonlinear optical response of distorted benzene," J. Phys. Chem. 99, 11061-11066 (1995).

${ }^{4}$ V. Buch, E. Gershgoren, H. Zabrodsky Hel-Or, and D. Avnir, "Symmetry loss as a criterion for cluster melting, with application to (D2)13," Chem. Phys. Lett. 247, 149-153 (1995).

${ }^{5}$ H. Zabrodsky and D. Avnir, "Continuous symmetry measures. 4. Chirality,” J. Am. Chem. Soc. 117, 462-473 (1995).

${ }^{6}$ G. Gilat and Y. Gordon, "Geometric properties of chiral bodies," J. Math. Chem. 16, 37-48 (1994).

${ }^{7}$ J. Maruani, G. Gilat, and R. Veysseyre, "Chirality limits of convex bodies," Compt. Rend. Acad. Sci. Paris 319, Sér. II, 1165-1172 (1994).

${ }^{8}$ N. Weinberg and K. Mislow, "Distance functions as generators of chirality measures," J. Math. Chem. 14, 427-450 (1993).

${ }^{9}$ Z. Zimpel, "A geometrical approach to the degree of chirality and asymmetry," J. Math. Chem. 14, 451-465 (1993).

${ }^{10}$ J. Maruani and P. G. Mezey, "From symmetry to syntopy: a fuzzy-set approach to quasi-symmetric systems," J. Chim. Phys. 87, 1025-1047 (1990).

${ }^{11}$ M. Petitjean, "Calcul de chiralité quantitative par la méthode des moindres carrés," Compt. Rend. Acad. Sci. Paris, Sér. IIc 2, 25-28 (1999).

${ }^{12}$ M. Petitjean, "About second kind continuous chirality measures. 1. Planar sets," J. Math. Chem. 22, 185-201 (1997).

${ }^{13}$ M. S. Srivastava and C. G. Khatri, An Introduction to Multivariate Statistics (Elsevier, New York, 1979), Chap. 3.

${ }^{14}$ M. S. Bartlett, "The effect of standardization on a $\chi^{2}$ approximation in factor analysis," Biometrika 38, 337-344 (1951).

${ }^{15}$ F. J. Schuurmann, P. R. Krishnaiah, and A. K. Chattopadhyay, "'On the distributions of the ratios of the extreme roots to the trace of the Wishart matrix," J. Multivariate Anal. 3, 445-453 (1973).

${ }^{16}$ J. R. Hurley and R. B. Cattell, "The Procrustes Program: Producing direct rotation to test a hypothesized factor structure," Behav. Sci. 7, 258-262 (1962).

${ }^{17}$ G. H. Golub and C. F. Van Loan, Matrix Computations (Johns Hopkins University Press, Baltimore, 1985), Secs. 8.1 and 12.4.

${ }^{18}$ A. D. McLachlan, "Rapid comparison of protein structures," Acta Crystallogr. Sect. A: Cryst. Phys., Diffr., Theor. Gen. Crystallogr. 38, 871-873 (1982).

${ }^{19}$ M. J. Sippl and H. Stegbuchner, "Superposition of three-dimensional objects: A fast and numerically stable algorithm for the calculation of the matrix of optimal rotation," Comput. Chem. 15, 73-78 (1991).

${ }^{20}$ R. Diamond, "A note on the rotational superposition problem," Acta Crystallogr., Sect. A: Found. Crystallogr. 44, 211-216 (1988).

${ }^{21}$ R. Deheuvels, Formes Quadratiques et Groupes Classiques (Presses Universitaires de France, Paris, 1981), p. 375.

${ }^{22}$ J. H. Wilkinson, The Algebraic Eigenvalue Problem (Clarendon, Oxford, 1965), Secs. 39-41. 